\title{
Solar Coronal Abundances of Rare Elements Based on Solar Energetic Particles
}

\author{
C. M. S. Cohen*, R. A. Mewaldt*, E. R. Christian ${ }^{\dagger}$, A. C. Cummings*, R. A. Leske*, \\ P. L. Slocum**, E. C. Stone* ${ }^{*}$ T. T. von Rosenvinge ${ }^{\dagger}$ and M. E. Wiedenbeck** \\ ${ }^{*}$ California Institute of Technology, MC 220-47, Pasadena, CA 91125 \\ ${ }^{\dagger}$ NASA/Goddard Space Flight Center, Code 661, Greenbelt, MD 20771 \\ ** Jet Propulsion Laboratory, 4800 Oak Grove Dr., Pasadena, 91109
}

\begin{abstract}
Although solar energetic particle (SEP) abundances vary from event to event, it has been shown that by accounting for these variations it is possible to use SEP data to obtain reliable estimates of elemental abundances for the solar corona. We analyze $\sim 20$ to $65 \mathrm{MeV} /$ nucleon measurements from the Solar Isotope Spectrometer on ACE in large SEP events observed from November 1997 to January 2001 to obtain new values of the average $\mathrm{SEP}$ composition of rare species, $\mathrm{P}, \mathrm{Cl}, \mathrm{K}, \mathrm{Ti}, \mathrm{Mn}, \mathrm{Cr}, \mathrm{Co}, \mathrm{Cu}$, and $\mathrm{Zn}$, which have had limited statistical accuracy in SEPs in the past. The measured SEP abundances are compared with other sources of solar-system composition data.
\end{abstract}

\section{INTRODUCTION}

It is clear that the composition of matter in many regions of the corona differs significantly from that of the photosphere. The first ionization potential (FIP) of the elements is commonly used to organize the differences, resulting in a step-like function where elements with FIPs less than $\sim 10 \mathrm{eV}$ are enhanced in the corona over elements with higher FIPs when compared to photospheric abundances (see, e.g., [1]). Although the abundances of nearly all the elements from $\mathrm{He}$ to $\mathrm{Ga}$ are relatively well known for the photosphere this is not the case for the solar corona. Because the coronal conditions are typically far from local thermal equilibrium, calculations of ionization equilibrium are difficult and significantly affect the accuracy of spectroscopically measured abundances [1]. Additionally, the charge state distribution of most elements is broad in the hot corona which results in the abundance of a particular element being distributed over many charge states, often yielding weaker spectral lines. This makes spectroscopic measurements of the abundance of elements such as $\mathrm{P}, \mathrm{Cl}, \mathrm{Co}$, and $\mathrm{Mn}$ particularly difficult. Thus, another way of determining these rare abundances is needed.

Although the FIP-fractionation is understood to be a ion-neutral separation process that occurs in the chromosphere/transition region [2], the specific mechanism involved is not known. In fact, arguments have been put forth that the elements' first ionization time (FIT) is a better organizing parameter [3]. Further, it has been observed that the FIP step height is not the same everywhere in the corona. A large step height has been inferred from the $\mathrm{Ne} / \mathrm{Mg}$ ratio measured in polar plumes and solar active regions [4] while the same ratio in coronal holes and the $\mathrm{Si} / \mathrm{C}$ ratio measured over sunspots indicate a small step height in these regions $[5,6]$. Thus deriving the elemental composition of the corona from that of the photosphere is not a simple matter.

Abundances measured in the solar wind are known to be similar to those obtained spectroscopically in the corona and so are a natural proxy for coronal composition [1]. Unfortunately, there are no measurements of the rare elements such as those mentioned above, although the first solar wind measurement of $\mathrm{Cr} / \mathrm{Fe}$ was recently made using the MASS instrument on SoHO [7]. It is possible that the relative abundances of $\mathrm{Mn}, \mathrm{Ni}$ and $\mathrm{Zn}$ will follow from the same data set in the near future (F. Ipavich, private communication), but currently such abundances are unavailable for the solar wind.

Solar energetic particle (SEP) events are believed to arise from two distinct origins [8]. Impulsive events contain flare accelerated material, typically last for hours and tend to be smaller in intensity than gradual events. Gradual events contain material that is accelerated out of the corona and solar wind by shocks driven by coronal mass ejections and last for days. Given their origin, it is natural to expect the composition of gradual SEP events to be similar to that of the corona and perhaps a useful measure

CP598, Solar and Galactic Composition, edited by R. F. Wimmer-Schweingruber

(C) 2001 American Institute of Physics 0-7354-0042-3/01/\$18.00 




FIGURE 1. Hourly-averaged intensities of 14 MeV/nucleon oxygen from launch in August 1997 to the beginning of 2001 .

of the coronal composition.

However, the elemental composition of gradual SEP events is known to vary from event to event due to transportation/acceleration effects which often can be characterized as a charge-to-mass $(\mathrm{Q} / \mathrm{M})$ dependent fractionation [9]. The effect of the $\mathrm{Q} / \mathrm{M}$ fractionation on the elemental composition can be accounted for to obtain an average coronal composition. Garrard and Stone (hereafter G\&S) [10] empirically corrected for the Q/M fractionation by comparing the measured abundances of lowFIP elements to photospheric abundances as a function of $\mathrm{Q} / \mathrm{M}$ and fitting a power law to the data. Reames [8] states that the event-to-event variation in the $\mathrm{Q} / \mathrm{M}$ fractionation tend to cancel out when many SEP events are averaged together resulting in an SEP composition that is representative of the average corona.

Additionally, while the FIP fractionation apparent in the spectroscopic coronal abundances is present in SEP events, the degree of fractionation varies from event to event $[11,12]$. Since the FIP fractionation is an ionneutral separation process and the SEPs are all ions, the variations observed in SEP events are reflections of the corona itself rather than a result of the acceleration/transport of the energetic particles. However, it is unclear how to directly relate the observed variation in FIP fractionation in SEP events to that observed spectroscopically on the Sun. Thus, we are left with the hope that averaging over many SEP events will result in a FIP step height that is 'typical' of the corona.

Although there are significant difficulties in using SEP data to obtain the composition of the solar corona, many rare elements that are not available through spectroscopy or the solar wind instruments can be measured with reasonable accuracy. With the increased sensitivity of today's energetic particle detectors, it is possible to measure the rare elements with an accuracy that is much improved over previous studies. Additionally, solar cycles are known to differ in character and analyzing the composition of events from solar cycle 23 is useful in furthering our understanding of both the solar corona and solar activity and their variations. 


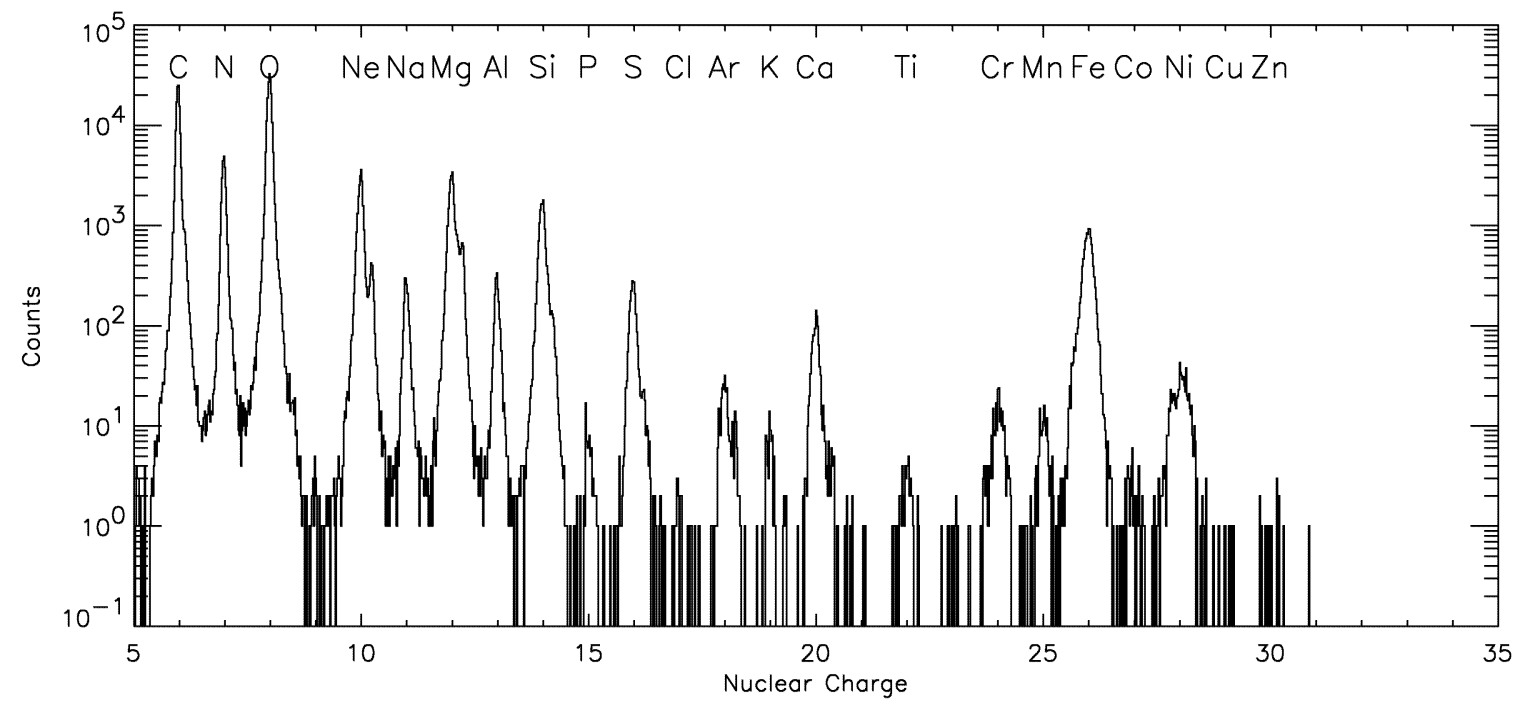

FIGURE 2. Histogram of events as a function of nuclear charge for particles stopping in the 3rd stack detector of SIS (corresponding to $\sim 19 \mathrm{MeV} /$ nucleon for oxygen).

\section{INSTRUMENTATION AND DATA ANALYSIS}

The data presented here are from the Solar Isotope Spectrometer (SIS; [13]) on the ACE spacecraft. The SIS sensor contains two identical telescopes, each of which has two position-sensing matrix detectors followed by a stack of large-area silicon detectors. The matrix detectors allow the particle trajectories to be determined, which combined with the deposited energies measured by the stack detectors allow accurate determinations to be made of nuclear charge, $Z$, mass, $M$, and total kinetic energy, $E$. The large geometry factor $\left(\sim 38 \mathrm{~cm}^{2}\right.$-sr) of SIS is key in obtaining statistically significant measurements of the rare elements that are the focus of this study.

We combine the data from 27 large SEP events which occurred between November 1997 and January 2001 (Figure 1). Combining the data makes it possible to identify statistically significant peaks in a nuclear-charge histogram which correspond to rare elements, something not possible on an event-by-event basis. Peaks for $\mathrm{P}, \mathrm{Cl}$, $\mathrm{K}, \mathrm{Ti}, \mathrm{Cr}, \mathrm{Mn}, \mathrm{Co}, \mathrm{Cu}$, and $\mathrm{Zn}$ can be clearly identified in Figure 2 with little apparent contamination from neighboring, more abundant elements.

The 27 events examined varied not only in intensity but in composition, spectral shape, and in FIP and Q/M fractionation. By taking ratios of elements with similar FIP and Q/M values, the fractionation effects can be minimized. Any residual fractionation is further reduced by combining the data from many events, resulting in an 'average' abundance that is reasonably representative of a global average of the solar corona. Such an averaging technique was used by Reames [14] to infer coronal abundances from 5-12 MeV/nucleon energetic particles.

From the combined data set the differential-intensity spectrum of each element is determined. At several tens of $\mathrm{MeV} /$ nucleon the contribution of galactic cosmic rays (GCRs) can be substantial and must be subtracted from the spectra before calculating abundances. The GCR spectra are determined by analyzing a data set that consists of 252 days of low solar activity (as defined by the 1.0-4.75 MeV/nucleon daily-averaged proton flux measured by the Electron, Proton, Alpha Monitor on ACE being less than $\left.0.16\left(\mathrm{~cm}^{2} \text {-sec-sr-MeV }\right)^{-1}\right)$. The lowsolar-activity days were spread throughout the November 1997 to January 2001 time period to account for variations in the GCR intensities due to changing levels of solar modulation during this time period. Contributions from anomalous cosmic rays (ACRs) are present and are also accounted for through the low-activity data.

Both the SEP spectra and the low-activity spectra for $\mathrm{P}, \mathrm{Cl}, \mathrm{K}, \mathrm{Ti}, \mathrm{Cr}, \mathrm{Mn}, \mathrm{Co}, \mathrm{Ni}, \mathrm{Cu}$, and $\mathrm{Zn}$ are shown in Figure 3. For $\mathrm{Co}, \mathrm{Cu}$ and $\mathrm{Zn}$ there are insufficient statistics in the low-activity data set to obtain intensities for all energy bins. For these elements the low-activity spectra are estimated by scaling the Fe low-activity spectrum by the $\mathrm{Co} / \mathrm{Fe}, \mathrm{Cu} / \mathrm{Fe}$, and $\mathrm{Zn} / \mathrm{Fe}$ GCR abundances as measured by the Cosmic Ray Isotope Spectrometer on ACE (M. Wiedenbeck, private communication). These spectra are shown in Figure 3 as solid lines and are in agreement with the available intensities measured with SIS. After subtracting the low-activity spectra, the re- 

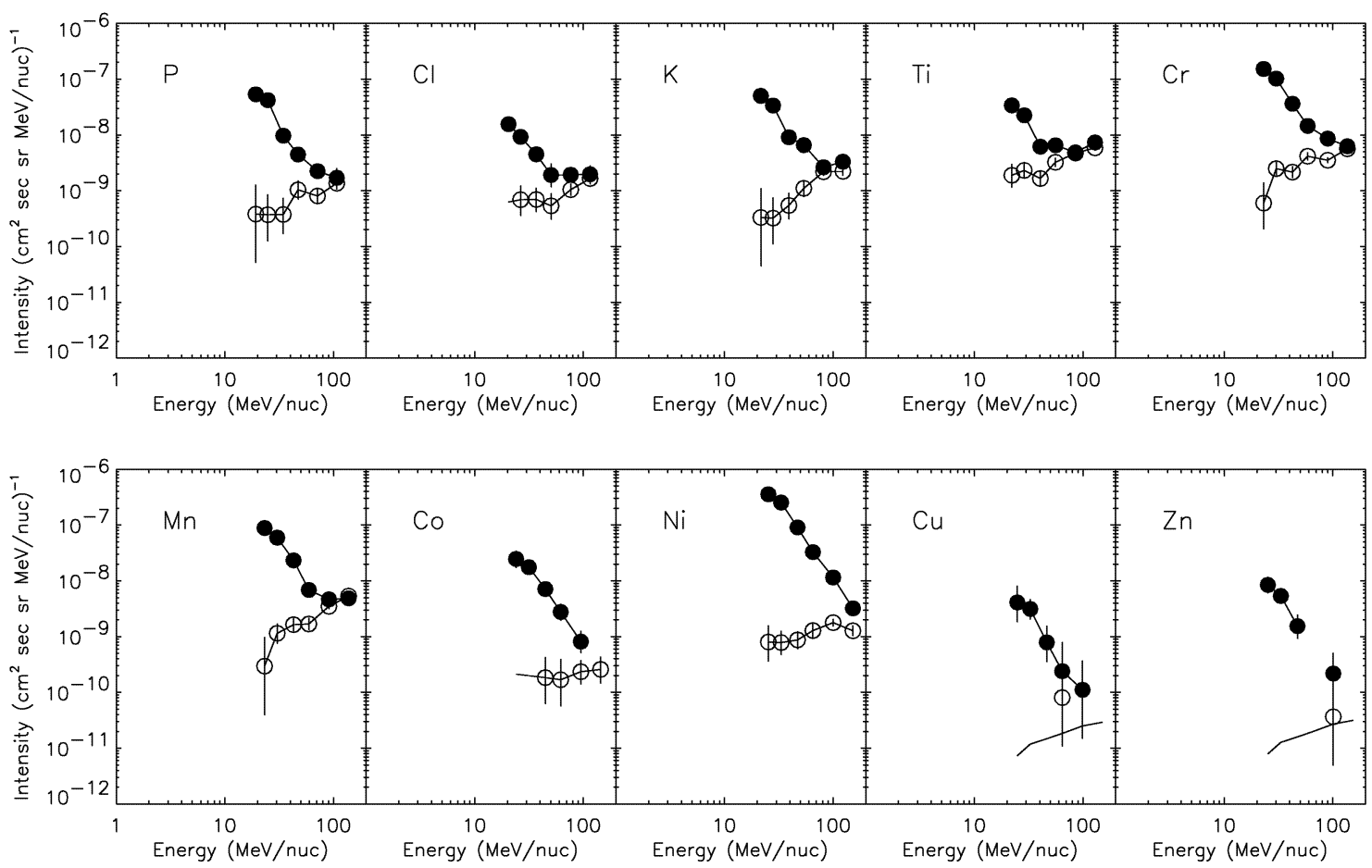

FIGURE 3. Spectra for different elements. Filled circles are SEP data, open circles are low-activity data. The solid lines are approximations of the low-activity spectra, obtained by scaling the Fe low-activity spectrum by the GCR abundances relative to Fe.

sulting corrected spectra are integrated from 20 to 65 $\mathrm{MeV} /$ nucleon to obtain relative abundances. The high end of this energy range was chosen such that the GCR corrections were not substantial (less than $50 \%$ contribution) while the low end was chosen based on where the nuclear charge resolution of the instrument was adequate for resolving the rare elements of interest.

\section{RESULTS}

The abundances of $\mathrm{P}, \mathrm{Cl}, \mathrm{K}, \mathrm{Ti}, \mathrm{Cr}, \mathrm{Mn}, \mathrm{Co}, \mathrm{Ni}, \mathrm{Cu}$, and $\mathrm{Zn}$ (relative to $\mathrm{Si}, \mathrm{Ca}$ or $\mathrm{Fe}$ ) are presented in Figure 4 (and in Table 1). For comparison the SEP measurements of Reames (hereafter DVR) [14] and Breneman and Stone (hereafter B\&S) [9] are given as well. The DVR values were obtained from a combined data set of 49 gradual $\mathrm{SEP}$ events and measured over 5-12 MeV/nucleon, while the $\mathrm{B} \& \mathrm{~S}$ values were from $3.5-50 \mathrm{MeV} /$ nucleon SEPs. The $B \& S$ values were then used by G\&S to derive coronal abundances by explicitly correcting for the apparent $\mathrm{Q} / \mathrm{M}$ fractionation by fitting a power law in $\mathrm{Q} / \mathrm{M}$ to the low-FIP elements. These results are also given in Figure 4 and Table 1 along with the abundance ratios as measured in the photosphere and meteorites [15]. The uncertainty in the fractionation correction is included in the uncertainties plotted for the G\&S values, whereas the uncertainties for the other SEP values are statistical only. By examining ratios of neighboring elements, the $\mathrm{Q} / \mathrm{M}$ fractionation is minimized, as can be seen from the small differences between the B\&S observed SEP and G\&S SEP-derived coronal values.

Generally all the SEP abundances agree well with each other except for $\mathrm{Cr} / \mathrm{Fe}$ and $\mathrm{Ni} / \mathrm{Fe}$. In the case of $\mathrm{Mn}$ and $\mathrm{Cu}$ there are no reported values by DVR and values with large uncertainties from $B \& S$. There are no other SEP values for Co. While the $\mathrm{Ni} / \mathrm{Fe}$ is higher in this work than that obtained by DVR and B\&S, it is close to the photospheric and meteoritic values. $\mathrm{Cr} / \mathrm{Fe}$ and $\mathrm{Co} / \mathrm{Fe}$, however, are $30 \%$ and $50 \%$ higher than the meteoritic abundances. It does not appear from Figure 2 (and from examination of a similar histogram of particles stopping in the second stack detector; not shown) that contamination from the $\mathrm{Fe}$ peak is the cause of this discrepancy as the peaks are well separated and there is little apparent background.

By choosing the ratios appropriately, the FIP fractionation is not a significant contribution to any difference between the photospheric (or meteoritic) and SEP abundances. Note that since P has a FIP value of $10.5 \mathrm{eV}$ it, like $\mathrm{S}$, is a transition element which does not appear to 

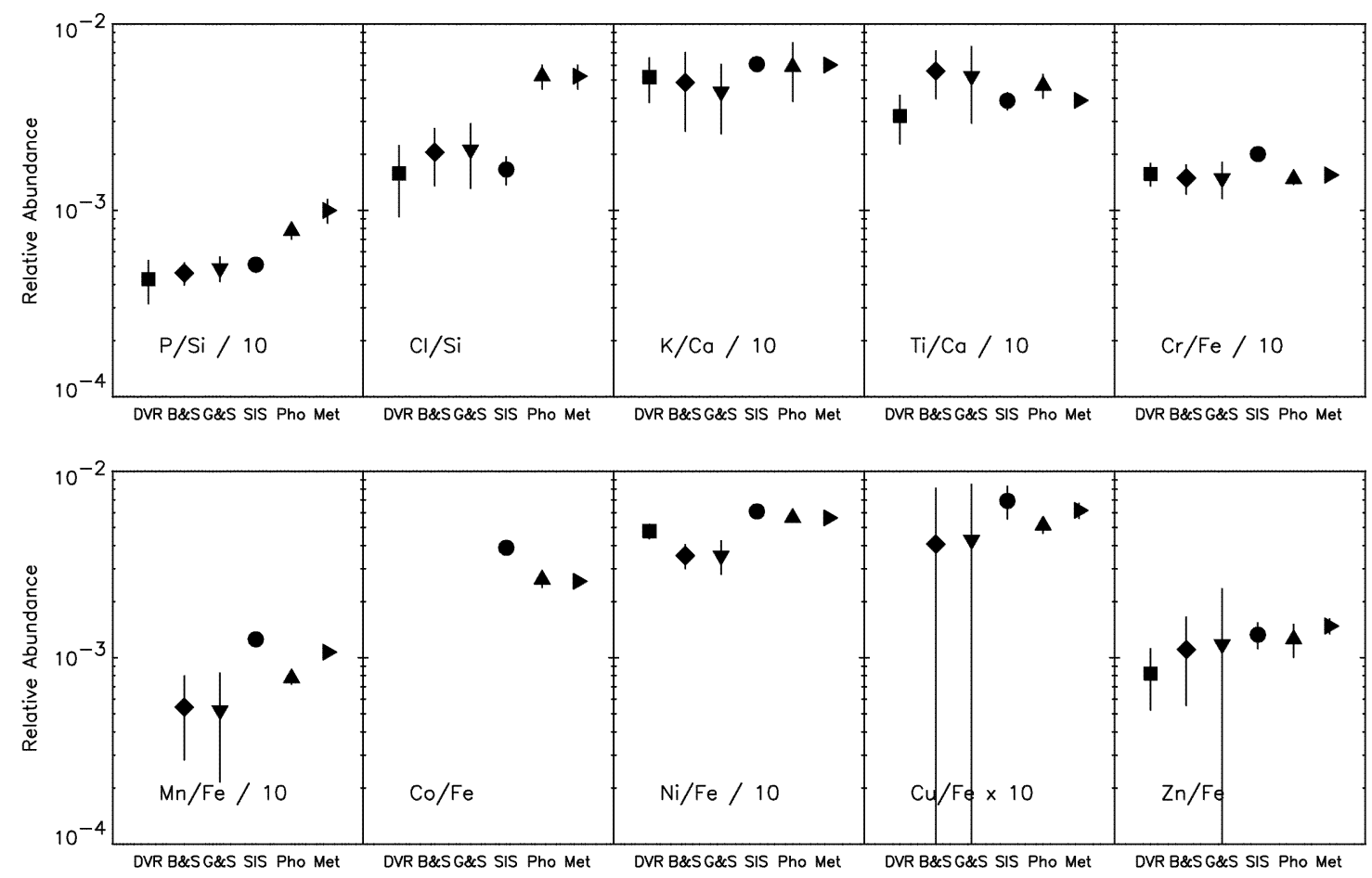

FIGURE 4. Resulting elemental ratio abundances for 20-65 MeV/nucleon as compared to SEP measurements by Reames (DVR) [14] and Breneman and Stone (B\&S) [9]; SEP-derived coronal abundances by Garrard and Stone (G\&S) [10]; and photospheric (Pho) and meteoritic (Met) abundances by Grevesse and Sauval [15].

belong to either the high-FIP or low-FIP element groups, and so the $\mathrm{P} / \mathrm{Si}$ ratio is only partially depleted relative to photospheric values. The $\mathrm{Cl} / \mathrm{Si}$ ratio is another exception in that $\mathrm{Cl}$ is a high-FIP element while $\mathrm{Si}$ is a lowFIP one. A more appropriate ratio would be $\mathrm{Cl} / \mathrm{Ar}$ (two high-FIP elements), but both photospheric and meteoritic abundances for Ar are unavailable (note, the Ar entry in most tables for photospheric abundances is based on the Ar measurements from solar wind or SEPs [15]).

A modification of the analysis is required to extend the present study to a comprehensive one of all the elements, C-Zn, measured by SIS. By combining the data from SEP events of significantly different intensities, the resulting data set is skewed towards the larger intensity events. Such an averaging technique does not seem appropriate for element ratios that vary significantly between events due to $\mathrm{Q} / \mathrm{M}$ or FIP fractionation effects.

Certainly such weighting issues are less of a concern when there is little event-to-event variability in the SEP composition. Evaluation of the variation of abundances as a function of energy has shown that the event-toevent abundance variation at $1 \mathrm{MeV} /$ nucleon is less than it is above $20 \mathrm{MeV} /$ nucleon [16]. Thus the averaging technique of creating and analyzing a combined data set from many SEP events may be more appropriate at energies near $1 \mathrm{MeV} /$ nucleon.

In order to derive a complete set of meaningful coronal abundances from several tens of $\mathrm{MeV} /$ nucleon SEP data, it may be necessary to make event-specific $\mathrm{Q} / \mathrm{M}$ fractionation corrections to the data prior to calculating average abundances. While this poses a statistical problem for the rare species a compromise may be possible. By characterizing each event by its $\mathrm{Q} / \mathrm{M}$ fractionation (possibly using the $\mathrm{Fe} / \mathrm{Si}$ ratio as a proxy [12]), events of similar fractionation can then be combined to increase statistical accuracy and then corrected for the $\mathrm{Q} / \mathrm{M}$ fractionation before taking an average of the results of different event groups. Such an averaging technique will be investigated in future work.

\section{CONCLUSIONS}

By combining the SIS data from 27 large SEP events, we obtain statistically accurate measurements of $\mathrm{P}, \mathrm{Cl}, \mathrm{K}, \mathrm{Ti}$, $\mathrm{Cr}, \mathrm{Mn}, \mathrm{Co}, \mathrm{Cu}$ and $\mathrm{Zn}$. These measurements are significant improvements over previous SEP determinations. Since these elements are extremely difficult to measure spectroscopically in the corona, SEP measurements are currently the best determined sample of coronal material. 
TABLE 1. Elemental Ratios Multiplied by 1000

\begin{tabular}{|c|c|c|c|c|c|c|c|}
\hline $\begin{array}{c}\text { Element } \\
\text { Ratio }\end{array}$ & $\begin{array}{l}\text { FIP } \\
(\mathbf{e V})^{*}\end{array}$ & Reames $^{\dagger}$ & $\begin{array}{l}\text { Breneman } \\
\text { and Stone }\end{array}$ & $\begin{array}{l}\text { Garrard } \\
\text { and Stone }\end{array}$ & $\begin{array}{l}\text { This } \\
\text { Work }^{\S}\end{array}$ & Photosphere & Meteoritic \\
\hline $\mathrm{P} / \mathrm{Si}$ & 10.49 & $4.28 \pm 1.12$ & $4.61 \pm 0.65$ & $4.89 \pm 0.75$ & $5.12 \pm 0.47$ & $7.76 \pm 0.75$ & $10.00 \pm 1.48$ \\
\hline $\mathrm{Cl} / \mathrm{Si}$ & 13.00 & $1.58 \pm 0.66$ & $2.05 \pm 0.70$ & $2.12 \pm 0.81$ & $1.66 \pm 0.29$ & $5.24 \pm 0.78$ & $5.25 \pm 0.78$ \\
\hline $\mathrm{K} / \mathrm{Ca}$ & 4.34 & $51.89 \pm 14.15$ & $48.53 \pm 22.06$ & $43.25 \pm 17.56$ & $60.87 \pm 4.78$ & $58.87 \pm 20.56$ & $60.26 \pm 2.84$ \\
\hline $\mathrm{Ti} / \mathrm{Ca}$ & 6.82 & $32.08 \pm 9.43$ & $55.88 \pm 16.18$ & $52.48 \pm 23.20$ & $38.79 \pm 4.22$ & $46.74 \pm 6.97$ & $38.90 \pm 1.83$ \\
\hline $\mathrm{Cr} / \mathrm{Fe}$ & 6.77 & $15.67 \pm 2.24$ & $14.91 \pm 2.71$ & $14.86 \pm 3.30$ & $20.06 \pm 0.85$ & $14.76 \pm 1.08$ & $15.49 \pm 0.36$ \\
\hline $\mathrm{Mn} / \mathrm{Fe}$ & 7.44 & & $5.42 \pm 2.61$ & $5.68 \pm 3.34$ & $12.55 \pm 0.68$ & $7.76 \pm 0.56$ & $10.72 \pm 0.25$ \\
\hline $\mathrm{Co} / \mathrm{Fe}$ & 7.86 & & & & $3.88 \pm 0.31$ & $2.63 \pm 0.26$ & $2.57 \pm 0.06$ \\
\hline $\mathrm{Ni} / \mathrm{Fe}$ & 7.64 & $47.76 \pm 4.48$ & $35.25 \pm 5.21$ & $35.24 \pm 7.32$ & $60.89 \pm 1.42$ & $56.23 \pm 1.31$ & $56.23 \pm 1.31$ \\
\hline $\mathrm{Cu} / \mathrm{Fe}$ & 7.73 & & $0.41 \pm 0.51$ & $0.47 \pm 0.75$ & $0.69 \pm 0.14$ & $0.51 \pm 0.05$ & $0.62 \pm 0.06$ \\
\hline $\mathrm{Zn} / \mathrm{Fe}$ & 9.39 & $0.82 \pm 0.30$ & $1.11 \pm 0.55$ & $1.18 \pm 1.31$ & $1.33 \pm 0.21$ & $1.26 \pm 0.26$ & $1.48 \pm 0.14$ \\
\hline
\end{tabular}

${ }^{*}$ FIP of numerator; FIP values for $\mathrm{Si}, \mathrm{Ca}$, and $\mathrm{Fe}$ are $8.12,6.09$, and $7.83 \mathrm{eV}$ respectively

${ }^{\dagger}$ from [14] average SEP abundances

** from [9] average SEP abundances

\# from [10] SEP-derived coronal abundances

$\S$ average SEP abundances

I from [15]

However, Q/M fractionation can significantly alter the SEP abundances from the original coronal composition and must be accounted for. At tens of $\mathrm{MeV} /$ nucleon energies this fractionation can vary substantially from event to event and may need to be corrected on an event-byevent basis. Balancing this with the need for statistical accuracy in obtaining the abundances of rare elements will be an aspect of future work. These studies will be aided by direct examination of the event-to-event variability for some elements such as Cr. In this work, we have dealt with these fractionation issues by examining abundance ratios of neighboring elements with similar FIP and Q/M values.

\section{ACKNOWLEDGMENTS}

This work was supported by NASA at Caltech (under grant NAG5-6912), Jet Propulsion Laboratory, and Goddard Space Flight Center.

\section{REFERENCES}

1. Meyer, J. P., Astrophys. J. Supp., 57, 173-204 (1985).

2. Henoux, J. C., Space Sci. Rev., 85, 215-226 (1998).

3. Geiss, J., Space Sci. Rev., 85, 241-252 (1998).

4. Widing, K., and Feldman, U., Astrophys. J., 416, 392-397 (1993).

5. Feldman, U., Space Sci. Rev., 85, 227-240 (1998).

6. Dere, K. P., Bartoe, J.-D. F., and Brueckner, G., Astrophys. J., 259, 366-371 (1982).

7. Paquette, J. A., Ipavich, F., Lasley, S., Bochsler, P., and P.Wurz, "The Relative Abundance of Chromium and Iron in the Solar Wind", in Proc. Joint SoHO-ACE Workshop
2001, AIP Conf. Proc., AIP, New York, 2001, this volume.

8. Reames, D. V., Richardson, I. G., and Barbier, L. M., Astrophys. J. Lett., 382, L43-L46 (1991).

9. Breneman, H. H., and Stone, E. C., Astrophys. J. Lett., 299, L57-L61 (1985).

10. Garrard, T. L., and Stone, E. C., Proc. 23rd Internat. Cosmic Ray Conf. (Calgary), 3, 384-387 (1993).

11. Garrard, T. L., and Stone, E. C., Adv. Space Res., 14, (10)589-(10)598 (1994).

12. Mewaldt, R. A., et al., "Variable Fractionation of Solar Energetic Particles According to First Ionization Potential", in Acceleration and Transport of Energetic Particles Observed in the Heliosphere: ACE 2000 Symposium, edited by R. A. Mewaldt et al., AIP Conf. Proc. 528, AIP, New York, 2000, pp. 123-126.

13. Stone, E. C., et al., Space Sci. Rev., 86, 357-408 (1998).

14. Reames, D. V., Adv. Space Res., 15, (7)41-(7)51 (1995).

15. Grevesse, N., and Sauval, A. J., Space Sci. Rev., 85, 161-174 (1998).

16. Mazur, J. E., Mason, G. M., Klecker, B., and McGuire, R. E., Astrophys. J., 404, 810-817 (1993). 